\title{
Analysis of PAPR Reduction using Partial Transmit Sequence (PTS) Technique in OFDM
}

\author{
Mohit Agrawal $^{1}$, S. K. Sriwas ${ }^{2}$, J.P. Sani ${ }^{3}$, M. Shukla ${ }^{4}$ \\ M. Tech Student, Department of Electronics and Communication Engineering, B.I.E.T. Jhansi, U.P., India ${ }^{1}$ \\ Assistant Professor, Department of Electronics and Communication Engineering, B.I.E.T. Jhansi, U.P., India ${ }^{2}$ \\ Professor, Department of Electronics and Communication Engineering, B.I.E.T. Jhansi, U.P., India ${ }^{3}$ \\ Professor, Department of Electronics and Communication Engineering, HBTU Kanpur, India ${ }^{4}$
}

\begin{abstract}
From a current scenario demand of high speed multimedia data services increase day by day. Orthogonal Frequency Division Multiplexing (OFDM) is one of the important multi carrier systems and it is famous because it allow large no of subcarriers, high speed data rate and universal coverage with high speed mobility. OFDM is considerably degrades by peak-to-average-power ratio (PAPR).There are many scheme are developed to reduce the PAPR in OFDM system. In this paper we use Partial Transmit Sequence (PTS)technique to decrease the PAPR and simulation data of this technique is done by using Matlab.
\end{abstract}

Keywords: Peak-To-Average Power Ratio (PAPR), Orthogonal Frequency Division Multiplexing (OFDM), Partial Transmit Sequence (PTS), Quadrature Amplitude Modulation) QAM.).

\section{INTRODUCTION}

In an OFDM system many subcarrier signals are added in an IFFT operation as a result high peak is generated. Due to this reason OFDM systemhas a high PAPR (Peak-to-Average Power Ratio). In fact, the high PAPR is one of the most disadvantageous in the OFDM system, as it decreases the Signal-to-Quantization Noise Ratio (SQNR) of DAC and ADCand also degrading the performance of the power amplifier in the system[1]. In the uplink the PAPR problem is more significant since the efficiency of power amplifier is perilous due to the restricted battery power in a mobile terminal.

The major problem of the OFDM system is high PAPR of communicating signal in transmitter side which reduces the efficiency of the OFDM system when a non-linear HPA (High power amplifier) is used. Due to this reason researches develop the PAPR reduction technique to reduce PAPR at the transmitter side. Several techniques are present in the literature to minimize the PAPR like clipping\& filtering,Tone Reservation Selected Mapping (SLM), Interleaving Technique, Tone Injection (TI), Peak Windowing, DFT spreading,Partial Transmit Sequence [2-4].

The major impact of a high PAPR are:

1. Increased complexity in the ADC and DAC.

2. $\quad$ Reduced in efficiency of radio frequency (RF) amplifiers.

In this research PTS (Partial Transmit Sequence)techniqueis used in PAPR reduction. Simulation results are discussed and analysed.

\section{SYSTEM MODEL}

\section{A. Basic OFDM system}

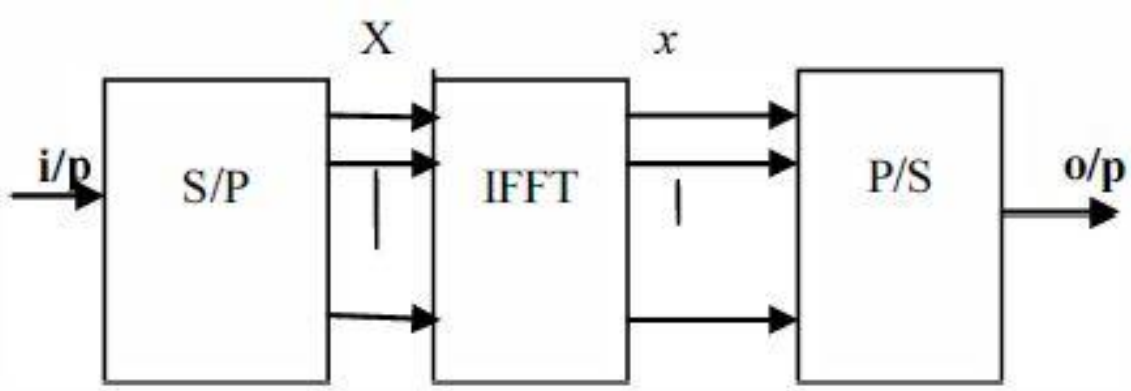

Fig.1 Basic Block Diagram of OFDM System 
Above shows the basic block diagram of OFDM system .The incoming data first passes through serialto parallel convertor and after conversion to parallel complex symbols of size ' $\mathrm{N}$ ' i.e. $\mathrm{X}=\left[\mathrm{x}_{0}, \mathrm{X}_{1}, \mathrm{X}_{2} \ldots \ldots \mathrm{x}_{\mathrm{N}-1}\right]^{\mathrm{T}}$, where ' $\mathrm{N}$ ' is total number of sub carriers used for parallel transmission then IFFT is taken for all the symbols. Output is denoted as

$$
\mathrm{x}(\mathrm{n})=\frac{1}{\sqrt{\mathrm{N}}} \sum_{\mathrm{k}=0}^{\mathrm{N}-1} \mathrm{X}_{\mathrm{k}} \mathrm{e}^{\frac{\mathrm{j} 2 \pi \mathrm{kt}}{\mathrm{N}}}, \quad 0 \leq \mathrm{n} \leq \mathrm{N}-1
$$

\section{B. PAPR in OFDM}

The OFDM signal is generated using input symbols $\mathrm{X}_{\mathrm{k}}$ is shown below where $\mathrm{n}$ is no of subcarriers as

$$
\mathrm{x}(\mathrm{n})=\frac{1}{\sqrt{\mathrm{N}}} \sum_{\mathrm{k}=0}^{\mathrm{N}-1} \mathrm{X}_{\mathrm{k}} \mathrm{e}^{\frac{\mathrm{j} 2 \pi \mathrm{kt}}{\mathrm{N}}}, \quad 0 \leq \mathrm{n} \leq \mathrm{N}-1
$$

The PAPR of OFDM signals $\mathrm{x}(\mathrm{n})$ is defined as the ratio between the maximum instantaneous power and its averagePower [5]. The PAPR (in $\mathrm{dB}$ ) of the transmitted OFDM signal can be defined as:

$$
\mathrm{PAPR}=\frac{\left.\max _{0 \leq \mathrm{n} \leq \mathrm{N}-1}\left[|\mathrm{x}(\mathrm{n})|^{2}\right]\right]}{\mathrm{E}\left(|\mathrm{x}(\mathrm{n})|^{2}\right)}
$$

Where $\mathrm{E}$ [.] is the expected value operator. In an OFDM system no of sub carriers are sum up togetherand it is called as anOFDM symbol. When large no of carriers added and due to this instantaneous power output at some point become greater than the mean power of system. Due to this major problem OFDM has high PAPR. High PAPR signals demands a large range of dynamic linearity from the analog circuits which usually results in costly devices and high power intake with lower performance. If we not able to decreasethe high PAPR, OFDM system could face major problem in practical applications.

PAPR is known as random variable because the data is coming as a input is random in nature. Therefore central limit theorem is used to calculate that how many times envelope of signal crosses level of signal. Aftercalculating the amplitude distribution of the OFDM signals, it is easy to calculate the probabilityof the instantaneous amplitude which is above the threshold and it is same for power also[6]. This isimplemented by calculating the CCDF (complementary cumulative distribution function) for different PAPR values as follows

$$
\mathrm{CCDF}=\operatorname{Pr}\left(\mathrm{PAPR}>\mathrm{PAPR}_{0}\right)
$$

The Cumulative Distribution Function (CDF) is one of the most regularly used parameters, which is used to measure the efficiency of any PAPR technique. The CCDF help in calculating the probability and we know that PAPR is easily described and analysed.

\section{OFDM SYSTEM WITH PTS METHOD}

PAPR reduction using PTS scheme is shown in figure, Xis called frequency domain vector is divided into various disjoint sub-blocks(v), which is denoted as $\{\mathrm{X}(\mathrm{v}), \mathrm{v}=0,1,2 \ldots \mathrm{N}-1\}$.

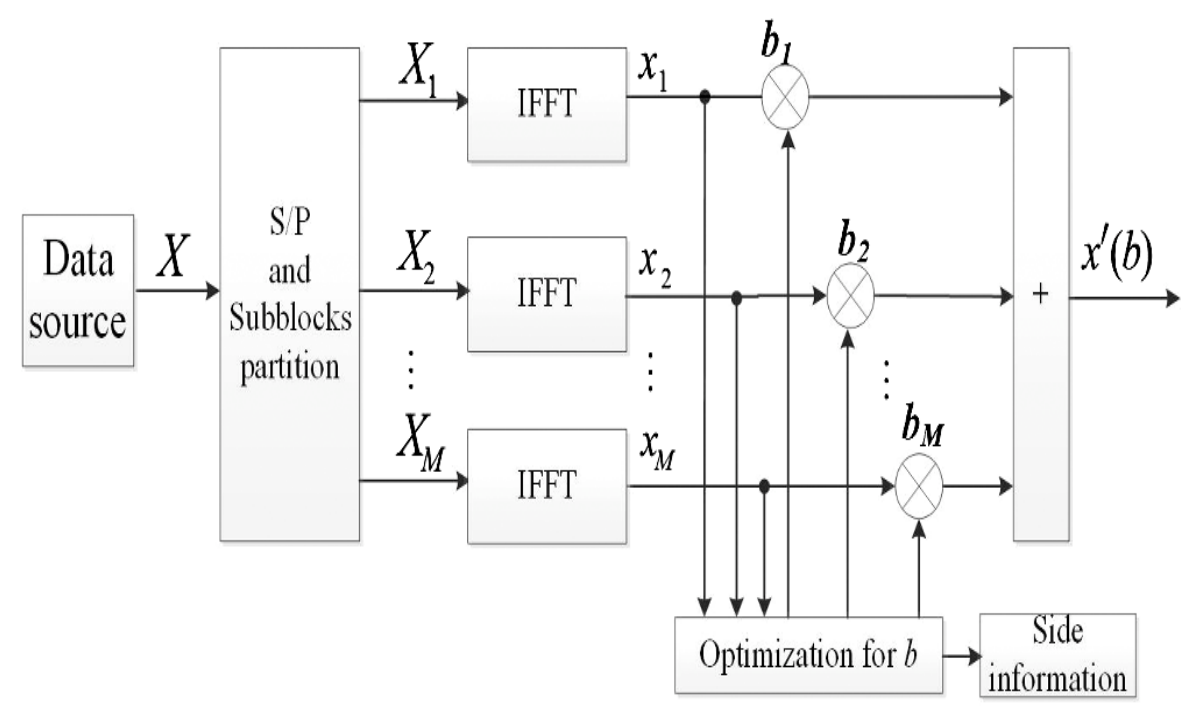

Fig.2 Block diagram of the optimal PTS scheme 
Hence,

$$
\mathrm{X}=\sum_{\mathrm{v}=1}^{\mathrm{V}} \mathrm{X}^{\mathrm{v}}
$$

Where $X^{v}=\left[X_{0}{ }^{v}, X_{1}{ }^{v} \ldots \ldots \ldots . . . X_{N-1}{ }^{v}\right], X_{k}{ }^{v}=X_{k}$ or 0 . Let $b=\left\{b_{v}=e^{j \theta}, v=1,2,3 \ldots . V\right\}$ be the set of phasefactors which are applied to the subblocks $\mathrm{X}(\mathrm{v})$. The transitory frequency domain signals are:

$\mathrm{X}^{\prime}=\sum_{\mathrm{v}=1}^{\mathrm{V}} \mathrm{b}_{\mathrm{v}} \mathrm{X}^{\mathrm{v}},\left(\mathrm{b}_{\mathrm{v}}=\mathrm{e}^{\mathrm{j} \theta}, \mathrm{v}=1,2,3 \ldots \mathrm{V}\right)$

Note that these partial categorizations are independently rotated by phase factors b. taking the IFFT and by using the linearity property of the IFFT, the time domain PTS can be denoted as:

$$
\mathrm{X}^{\prime}=\operatorname{IIFT}\left\{\mathrm{X}^{\prime}\right\}=\sum_{\mathrm{v}=1}^{\mathrm{V}} \mathrm{b}_{\mathrm{v}} \mathrm{X}^{\mathrm{v}}
$$

Select one appropriate factor combination $b_{\mathrm{V}}(\mathrm{v}=1,2, \ldots, \mathrm{V})$, by this we achieve optimum result. Thearrangement can be given by:

$$
\mathrm{b}=\underset{(\mathrm{b} 1, \mathrm{~b} 2, \ldots \mathrm{bv})}{\arg \min }\left(\max _{1 \leq \mathrm{n} \leq \mathrm{N}}\left|\sum_{\mathrm{v}=1}^{\mathrm{v}} \mathrm{b}_{\mathrm{v}} \mathrm{X}_{\mathrm{v}}\right|^{2}\right)
$$

$\operatorname{argmin}(\cdot)$ is the ruling condition that output is the minimum value of function. After calculating the optimum phase vectorby this min PAPR signal is transmitted to the next level. For the optimum PAPR performance it is calculate aftercalculating WV-1 different combinations if the number of permissible phases weighting factors is equal to W. In thecalculating process of $b$ (phase weighting) different combination, large numbers of complex multiplications and addition are needed due to this computational complexity increase considerably[7].

In the sub optimal PTS approach, we only take binaryweighting factors (i.e., 1 and -1). After dividing the input data block into Wclusters. As a first step, assume that $b_{v}=1$ for all $v$ and compute the PAPR of the added signal. Next, in second step first phase factor $(b=-1)$ and recalculate the resulting PAPR. If the calculated PAPR is lower than in the previous step, then save b1 as part of the final phase sequence, otherwise, b1 change to its previous value. The algorithm continues as above until all $\mathrm{V}$ possibilities have been explored [8]. In this way, the better phase factor can be found after only $\mathrm{V}$ times searching.

\section{SIMULATION RESULTS \& OBSERVATIONS}

In PTS, input data sequences are divided in to number of sub-blocks i.e. V=I, 2, 4 and 8 . In our simulation the total number of sub carriers used $\mathrm{N}=64$, Number of OFDM blocks $=3000$ and oversampling factor $\mathrm{L}=4$.

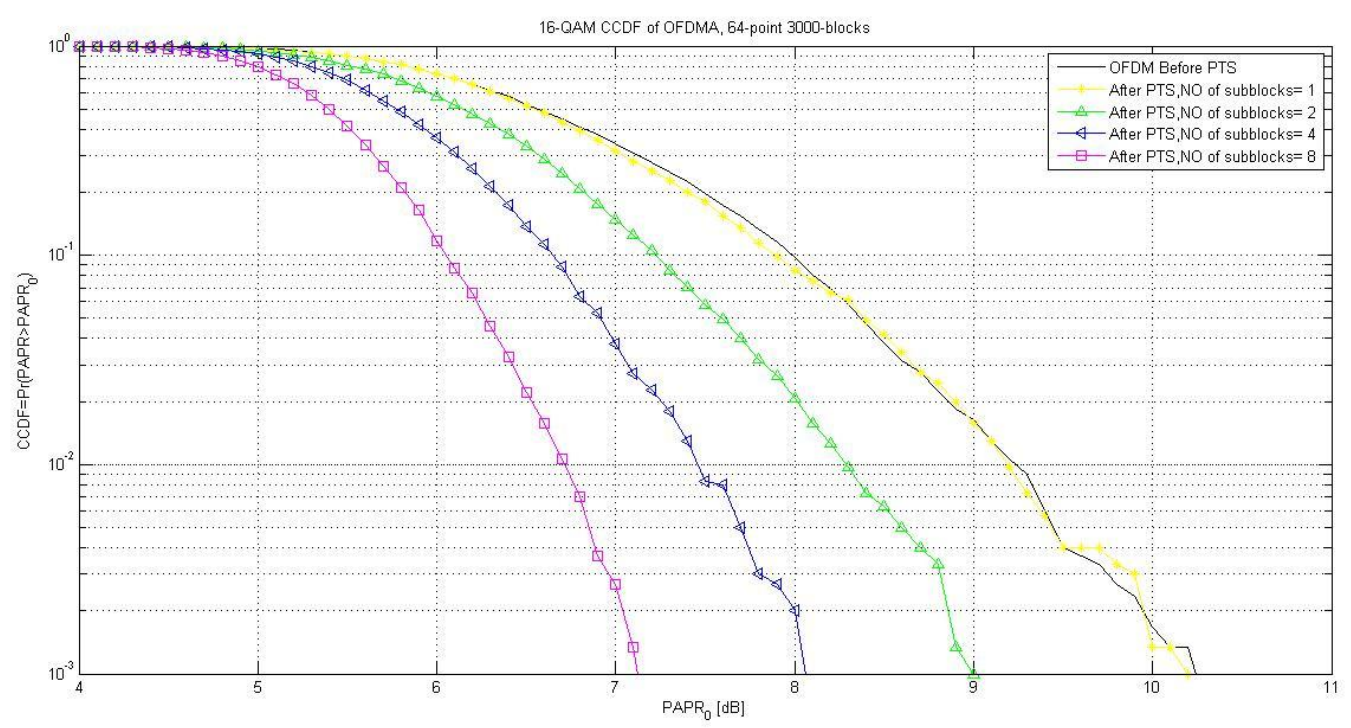

Fig. 3 CCOF of 16 QAM OFOM Signal 
Fig. 3 shows that the CCDF of 16-QAM OFDM signal with different number of sub blocks. As the number of sub block increases P APR decreases. So All the modulation methods PAPR is minimum when V=16.From the graph for V=16, PAPR is reduced by3.2dB than OFDM.

\section{CONCLUSION}

In this paper, we have analysed and shown the results for PAPR reduction of OFDM system using PTS when no of subblocks increases then PAPR reduction is more. As we see from the above simulated result at $v=1$ then PAPR is $10.3 \mathrm{~dB}$ approx. when we increase the no of subblocks such as $\mathrm{v}=8$ then PAPR is 7.1dB.

\section{REFERENCES}

[1] Foschini, Gerard J., and Michael 1. Gans. "On limits of wireless communications in a fading environment when using multiple antennas." Wireless personal communications 6.3 (1998): $311-335$

[2] R. Frederiksen, F. Prasad, "An overview of OFDM and related techniques towards development of future wireless multimedia communications", IEEE Radio and Wireless Conference Proceedings, 2002.

[3] S. Mohammady, R. Sidek, P.Varahram, M.Hamidon, and N. Sulaiman "Study of PAPR reduction methods in OFDM systems," in Advanced Communication Technology (ICACT), 2011 13th International Conference on 2011, pages 127- 130.

[4] S. H. Han and J. H. Lee, "An overview of peak-to-average power ratio reduction techniques for multicarrier transmission," Wireless Communications, IEEE, vol. 12, pp. 56-65, 2005.

[5] L.Xia, X.Yue, L.Shaqian, H.Kayama, and C.Yan, "Analysis of the performance of partial transmit sequences with different sequences with different sub block partitions", in communications, Circuits and Systemsm Proceedings, 2006 International Conference on, 2006 , pages 875876.

[6] Cimini Jr, Leonard 1., and Nelson R. Sollenberger. "Peak-to-average power ratio reduction of an OFDM signal using partial transmit sequences." Communications Lellers, IEEE 4.3 (2000): 86-88.

[7] Sharif, Masoud, Mohammad Gharavi-Alkhansari, and Babak Hossein Khalaj. "On the peak-to-average power of OFDM signals based on oversampling." Communications, iEEE Transactions on 51.1 (2003): 72-78.

[8] Wang, Jinkuan, Xin Song, and Bin Wang. "PAPR Reduction with Low Computational Complexity for OFDM Systems." Physics Procedia 33 (2012): 1401-1407. 